Western University

Scholarship@Western

Anatomy and Cell Biology Publications

Anatomy and Cell Biology Department

2013

\title{
Assessing the feasibility of time-resolved fNIRS to detect brain activity during motor imagery
}

\author{
Androu Abdalmalak \\ Western University, aabdalma@uwo.ca \\ Daniel Milej \\ Western University \\ Mamdou Diop \\ Western University \\ Lorina Naci \\ Western University \\ Adrian M. Owen \\ Western University, dpavich@uwo.ca \\ See next page for additional authors
}

Follow this and additional works at: https://ir.lib.uwo.ca/anatomypub

Part of the Anatomy Commons, and the Cell and Developmental Biology Commons

Citation of this paper:

Abdalmalak, Androu; Milej, Daniel; Diop, Mamdou; Naci, Lorina; Owen, Adrian M.; and St. Lawrence, Keith, "Assessing the feasibility of time-resolved fNIRS to detect brain activity during motor imagery" (2013). Anatomy and Cell Biology Publications. 21.

https://ir.lib.uwo.ca/anatomypub/21 
Authors

Androu Abdalmalak, Daniel Milej, Mamdou Diop, Lorina Naci, Adrian M. Owen, and Keith St. Lawrence 


\title{
Assessing the feasibility of time- resolved fNIRS to detect brain activity during motor imagery
}

\author{
Androu Abdalmalak \\ Daniel Milej \\ Mamadou Diop \\ Lorina Naci \\ Adrian M. Owen \\ Keith St. Lawrence
}




\title{
Assessing the feasibility of time-resolved fNIRS to detect brain activity during motor imagery
}

\author{
Androu Abdalmalak*a,b, Daniel Mileje, ${ }^{\mathrm{a}, \mathrm{b}}$, Mamdou Diop ${ }^{\mathrm{a}, \mathrm{b}}$, Lorina Naci ${ }^{\mathrm{c}}$, Adrian M. Owen ${ }^{\mathrm{c}}$, \\ Keith St. Lawrence ${ }^{\mathrm{a}, \mathrm{b}}$ \\ ${ }^{a}$ Department of Medical Biophysics, Western University, London, ON, Canada, N6A 5C1, \\ ${ }^{\mathrm{b}}$ Imaging Program, Lawson Health Research Institute, London, ON, Canada, N6A 4V2, \\ ${ }^{\circ}$ Brain and Mind Institute, Western University, London, ON, Canada, N6A 5B7
}

\begin{abstract}
Functional near-infrared spectroscopy (fNIRS) is a non-invasive optical technique for detecting brain activity, which has been previously used during motor and motor executive tasks. There is an increasing interest in using fNIRS as a brain computer interface (BCI) for patients who lack the physical, but not the mental, ability to respond to commands. The goal of this study is to assess the feasibility of time-resolved fNIRS to detect brain activity during motor imagery. Stability tests were conducted to ensure the temporal stability of the signal, and motor imagery data were acquired on healthy subjects. The NIRS probes were placed on the scalp over the premotor cortex (PMC) and supplementary motor area (SMA), as these areas are responsible for motion planning. To confirm the fNIRS results, subjects underwent functional magnetic resonance imaging (fMRI) while performing the same task. Seven subjects have participated to date, and significant activation in the SMA and/or the PMC during motor imagery was detected by both fMRI and fNIRS in 4 of the 7 subjects. No activation was detected by either technique in the remaining three participants, which was not unexpected due to the nature of the task. The agreement between the two imaging modalities highlights the potential of fNIRS as a BCI, which could be adapted for bedside studies of patients with disorders of consciousness.
\end{abstract}

Keywords: Time-resolved measurements, Functional near-infrared spectroscopy, Motor imagery

\section{INTRODUCTION}

Command-following using neuroimaging has been shown to be a means of detecting covert awareness in patients with disorders of consciousness ${ }^{1-3}$. Using motor imagery (i.e., imagining playing tennis), Owen et al. first introduced the concept of command following by regulating brain activity using $\mathrm{fMRI}^{4}$. Motor imagery such as imagining playing tennis activates brain regions such as the SMA and the $\mathrm{PMC}^{1,4,5}$. The results of that study showed that a patient who was clinically diagnosed as being in a vegetative state was able to follow commands by regulating her brain activity. This finding, along with other studies, highlight the ability of neuroimaging techniques to be used as tools to assess volition in brain injured patients ${ }^{6-8}$.

While fMRI provides high-resolution and global spatial coverage of brain activity, its cost and accessibility limit its use as a $\mathrm{BCI}^{2}$. A promising alternative is fNIRS, due to its portability and sensitivity to changes in cortical activity ${ }^{9,10}$. By placing optodes on the scalp and looking at the change in light absorption with respect to time, the change in the concentration of oxy- and deoxy-hemoglobin can be calculated ${ }^{11}$, which can be used to determine the presence of brain activity.

A major challenge with fNIRS is the decreased sensitivity to light absorption in the brain and, conversely, the greater sensitivity to oxygenation changes in the scalp. Various techniques have been put forward to overcome this issue, including multi-distance measurements on the scalp ${ }^{12-14}$, where the light detected at the largest source-detector distance will have a greater likelihood of reaching the brain. However, the accuracy of the multi-distance approach is hindered by its sensitivity to the skin's microvasculature ${ }^{15}$. An alternate technique is time-resolved (TR) fNIRS, which is based on the analysis of a distribution of time of flights (DTOFs) of photons that characterizes the broadening of a pulse of light

*aabdalma@uwo.ca; phone 15196466100 x 64186.

Clinical and Translational Neurophotonics; Neural Imaging and Sensing; and Optogenetics and

Optical Manipulation, edited by Steen J. Madsen, et. al., Proc. of SPIE Vol. 9690, 969002

(C) 2016 SPIE · CCC code: $1605-7422 / 16 / \$ 18 \cdot$ doi: $10.1117 / 12.2209587$ 
due to scattering in a turbid media ${ }^{16,17}$. Depth sensitivity is provided by the arrival times of photons, since late arriving photons have a greater likelihood of reaching the brain compared to early arriving photons that primarily interrogated the superficial layers. Using moment analysis ${ }^{18,19}$ or binning techniques ${ }^{10}$, the late arriving photons can be extracted and analyzed.

The purpose of this study was to assess the feasibility of TR-fNIRS to detect brain activity during motor imagery. A series of experiments were first conducted to assess the stability of the system and noise levels. The feasibility of fNIRS in detecting brain activity during motor imagery was tested by acquiring fNIRS and fMRI data from healthy subjects, and comparing the two modalities.

\section{METHODS}

\subsection{Instrumentation}

The TR-fNIRS system used in this study was designed and built in-house, and has been previously described in detail elsewhere ${ }^{20}$. The light source used in this system was a pulsed picosecond laser operating at $\lambda=830 \mathrm{~nm}$ (PicoQuant, Berlin, Germany) with a pulse repetition rate of $80 \mathrm{MHz}$. The laser power delivered to the subject's head was adjusted by a neutral density filter (NDC-50-4M, Thorlabs, Newton, NJ, United States) mounted in front of the laser head. Light pulses were coupled by an objective lens $(\mathrm{NA}=0.25$, magnification $=10 \mathrm{X}$, Olympus, Japan) into a $1.5 \mathrm{~m}$ long multimode bifurcated fiber ( $\phi=0.4 \mathrm{~mm}, \mathrm{NA}=0.22$, Fiberoptics Technology, Pomfret, Connecticut, United States). The emission fiber and four detection fibers were secured on the scalp using the fNIRS cap described in section 2.2. The diffusively reflected light from the surface of the subject's head was collected using four $2 \mathrm{~m}$ fiber bundles $(\phi=3.6 \mathrm{~mm}$ $\mathrm{NA}=0.55$, Fiberoptics Technology, Pomfret, Connecticut, United States) and delivered to the hybrid photo-multiplier tubes (PMA Hybrid 50, PicoQuant, Berlin, Germany). The HydraHarp 400 (PicoQuant, Berlin, Germany) timecorrelated single photon counting unit was used to build the DTOFs.

\section{2 fNIRS cap}

The holder used in this study is shown in Figure 1a. The holder consisted of one emission and four detection fibers (source-detector separation $r_{\mathrm{SD}}=3 \mathrm{~cm}$ ) arranged in a cross configuration with the emission fiber placed over the $\mathrm{FCz}$ position according to the 10-10 international system for EEG electrode placement. This cross configuration was chosen so that the forward scattered light propagating in the brain would interrogate the SMA and the PMC. The holder consisted of a base and a fiber bundle holder. Both parts were designed using SpaceClaim (SpaceClaim Corporation, United States) and 3D printed (TAZ 5, LulzBot, United States) in house using NinjaFlex 3D Flexible Printing Filament (Fenner Drives, Inc. United States). The diameter of the base was $1.5 \mathrm{~cm}$, which provides enough room to remove the hair, which was done using ultrasound gel and a $1 \mathrm{cc}$ syringe plunger. The fiber holder had a diameter of $1.48 \mathrm{~cm}$, which provided a tight fit to the base and prevented the fiber from moving on the surface of the head. Since NinjaFlex 3D is an extremely flexible material, a $0.5 \mathrm{~mm}$ thick copper sheet was glued to the bottom of the base to provide structural rigidity and to allow the holder to conform to the shape of the subject's head. Finally, $2 \mathrm{~mm}$ thick foam was glued to the copper sheet to prevent it from touching the scalp. The holder was held in place on the head using a 54/58 cm EEG cap (EASYCAP GmbH, Germany). Figure $1 b$ shows a subject wearing the EEG cap with the holder.

a)

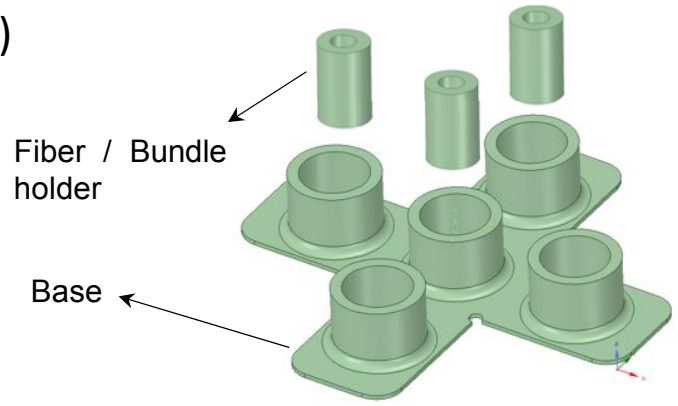

b)

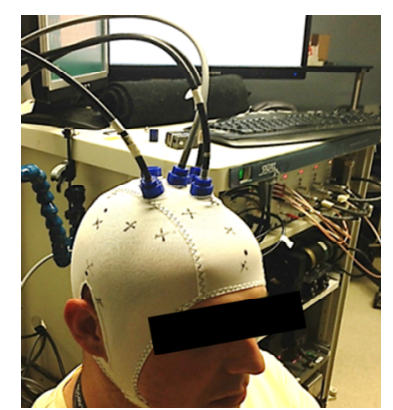

Figure 1. (a) Schematic of the fiber bundle holder and base used in this study. (b) Healthy subject wearing the fNIRS cap (only three channels shown). 


\subsection{Stability tests}

Before performing the activation studies, two sets of experiments were conducted in order to assess the stability of the system. First, the source and detector fibers were placed on the surface of a homogenous liquid phantom $\left(r_{\mathrm{SD}}=3 \mathrm{~cm}\right)$ filled with $20 \%$ Intralipid (Fresenius Kabi AG, Germany), water and ink solution ${ }^{21}$ in order to obtain optical properties similar to the human tissue $\left(\mu_{\mathrm{a}}=0.1 \mathrm{~cm}^{-1}, \mu_{\mathrm{s}}{ }^{\prime}=10 \mathrm{~cm}^{-1}\right)$. The DTOFs were then acquired every $0.2 \mathrm{~s}$ for five and half minutes.

Next, the signal stability and drift were assessed in vivo by placing the emission and detection fibers on the surface of the scalp using the fNIRS cap described above and asking the subjects to remain relaxed for the time of the experiment. The stability was measured on four subjects and once again, the DTOFs were acquired every $0.2 \mathrm{~s}$ for five and half minutes.

\subsection{Measurement procedure}

The motor imagery experimental protocol used in this study was previously described elsewhere ${ }^{5}$, which consisted of five cycles of 30 seconds alternating blocks of rest and tennis imagery, with a total experiment time of five and half minutes. The two cue words used in this study were "rest" and "tennis". When the subjects heard the word "tennis", they were asked to imagine swinging their arm as if they were trying to hit a tennis ball as hard as they can. They were asked to do so until they heard the word "rest" where they were asked to relax.

The experimental design consisted of the subjects first performing the motor imagery task in the MRI scanner, after which they were transferred to the optics lab where the fNIRS study was performed using the same experimental protocol. The fMRI data were obtained to verify the presence of activity and in order to confirm the fNIRS results.

Seven subjects ( 1 female, mean age 25 years, all right handed) with no history of neurological conditions have participated in the study to date. The study was approved by the University Research Ethics Board of the University of Western Ontario, and all volunteers provided written informed consent.

\subsection{Data acquisition}

The fMRI scans were conducted on the 3T Biograph mMR (Siemens Healthcare, Erlangen, Germany) at St. Joseph's Health Care, London, ON, Canada. A 32 channel receive only head coil was used, and high-resolution anatomical T1weighted MPRAGE images $\left(T R=2000 \mathrm{~ms}, \mathrm{TE}=2.98 \mathrm{~ms}, \mathrm{FA}=9^{\circ}\right.$, voxel size $\left.=1 \times 1 \times 1 \mathrm{~mm}\right)$ were first acquired. Next, the subjects were asked to perform the tennis imagery task as described above while $\mathrm{T} 2 *$-weighted images were continuously acquired $\left(\mathrm{TR}=3000 \mathrm{~ms}, \mathrm{TE}=30 \mathrm{~ms}, \mathrm{FA}=90^{\circ}\right.$, slice thickness $=3 \mathrm{~mm}$, voxel size $\left.=3 \times 3 \times 3 \mathrm{~mm}\right)$. The verbal cues were presented to the subjects using an MRI compatible headset.

The fNIRS data were acquired using the TR-NIRS system described in section 2.1. A series of DTOFs were acquired every $0.2 \mathrm{~s}$ at a resolution of $16 \mathrm{ps}$, for a total duration of five and half minutes.

\subsection{Data analysis}

The fMRI data were analyzed using the statistical parametric mapping (SPM8) software. Realignment was performed to correct for motion, and the images were normalized to the anatomical T1-weighted template in SPM. The images were smoothed using an $8 \mathrm{~mm}$ FWHM Gaussian kernel, and the time series were analyzed using the general linear model, with the condition (rest or tennis imagery) of each scan being defined. The data was then filtered using a high pass filter (cut off period of $128 \mathrm{~s}$ ). Finally, the data were corrected using a False Discovery Rate (FDR) statistical threshold with a p-value threshold of 0.05 .

The fNIRS data was analyzed using moment analysis. The number of photons $(N)$, mean time of flight $(<t>)$ and variance $(V)$ were calculated using ${ }^{18}$ :

$$
m_{k}=\int_{-\infty}^{\infty} t^{n} f(t) d t / \int_{-\infty}^{\infty} f(t) d t,
$$

where: $m_{k}$ is the $k^{\text {th }}$ normalized moment of a distribution $f(t)$ and $t$ represents time. 


\section{RESULTS}

\subsection{Stability tests}

The results of the conducted stability tests are presented in Figure 2. Changes in the statistical moments of the DTOF: number of photons $N$, mean time of flight $\langle t>$ and variance $V$ obtained from the phantom and from one subject are plotted against time. As expected, the signals obtained from the phantom are more stable and have fewer fluctuations than the in vivo signals. For the in vivo results, the stability of the signal improved with the higher the moments. Similar results were obtained for the other three subjects.

(a)

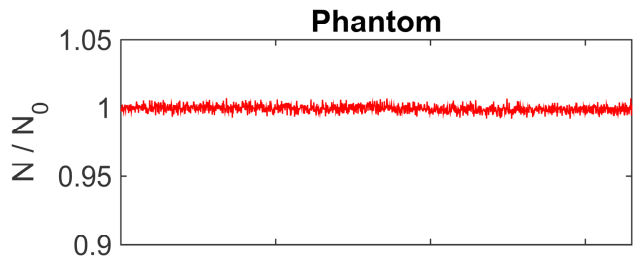

(b)

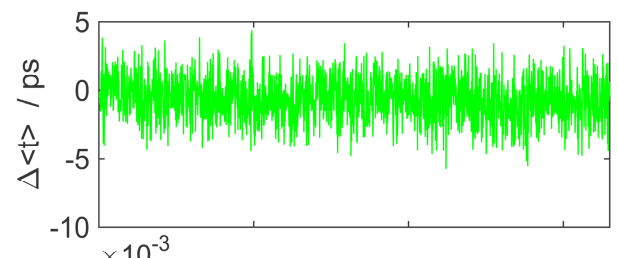

(c)

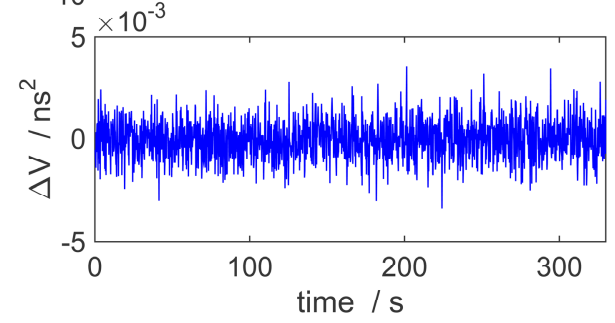

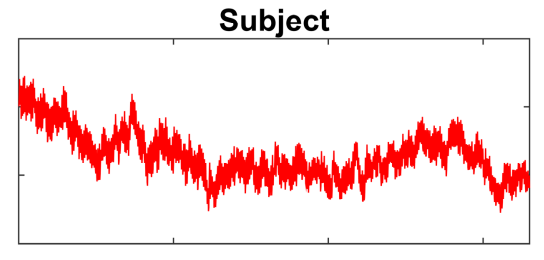
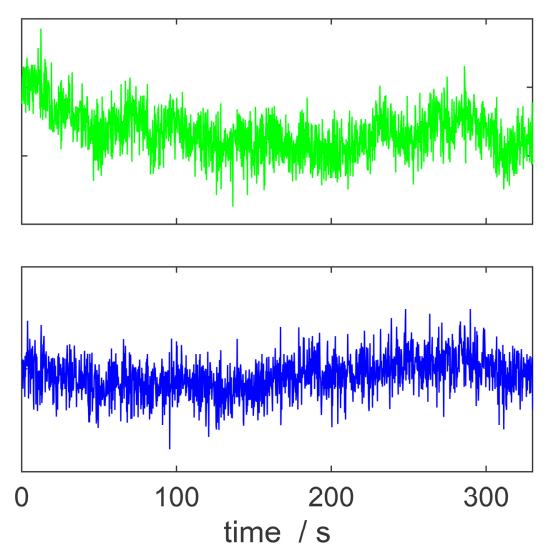

Figure 2. Changes in the statistical moments of the DTOFs plotted against time for one channel obtained from the tissuemimicking phantom and from one subject. (a) Normalized change in the number of photons $N$. The signals obtained were normalized by the initial value $N_{0}$ (mean value of $N$ for the first $10 \mathrm{~s}$ ). (b) Change in the mean time of flight $\Delta<t>$. (c) Change in variance $\Delta V$.

In order to determine the cause of the fluctuations in the statistical moments calculated from the in vivo signals, the time courses of the moments were normalized and frequency analysis was performed. Figure 3 shows the Fourier Transform of the normalized moments for one subject. A peak around $1.13 \mathrm{~Hz}$ corresponding to the heart rate of the subject can be observed for the number of photons (Figure 3a), which is not visible for either the mean time of flight or the variance.

(a)

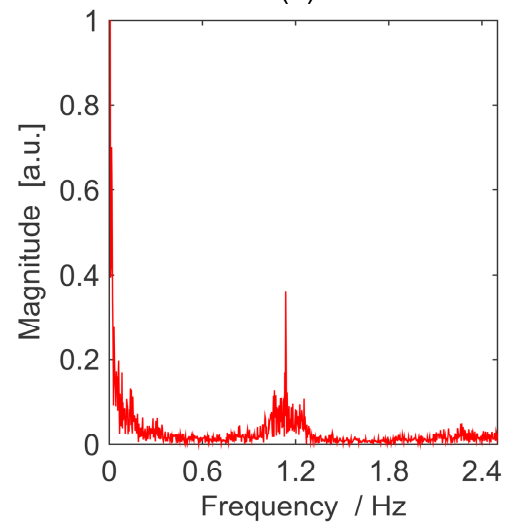

(b)

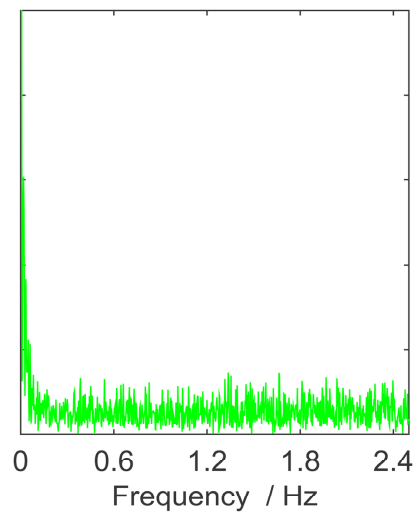

(c)

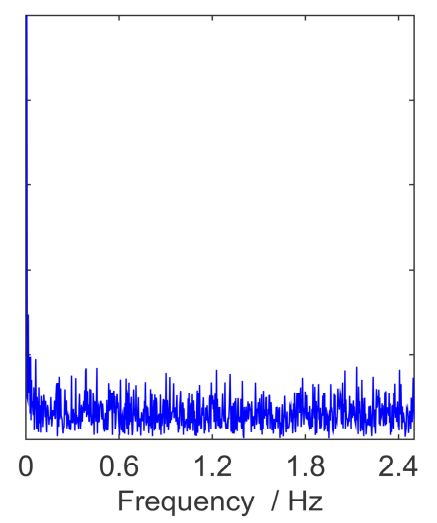

Figure 3. Fourier Transform for the (a) normalized number of photons (b) normalized mean time of flight and (c) normalized variance. 


\subsection{Motor imagery}

Activation in the SMA and PMC was detected by fMRI in 4 of the 7 subjects. Of these 4 subjects, activation was detected in both the SMA and the PMC in 3 subjects and only the PMC in the remaining subject. In agreement, brain activity was also observed by fNIRS in the same 4 subjects. In the remaining 3 subjects, no activation was detected by either technique.

Figure 4a shows the fMRI results obtained from one subject during rest and motor imagery overlaid on the single subject render template in SPM8. A band of activity can be observed across the PMC and SMA (FDR, 500 clusters, pvalue $<0.05$ ). In Figure $4 \mathrm{~b}$, the time courses of the normalized number of photon $N$ and the change in $<t>$ for one channel are presented. The fNIRS signals were filtered using a $4 \mathrm{~s}$ moving average window, and the data were detrended using a $4^{\text {th }}$ order polynomial in order to remove signal drift. It can be observed that during tennis imagery (indicated by the grey boxes), there is a decrease in the $N$ and the $<t>$, which returns to the baseline level once the task is completed.

(a)

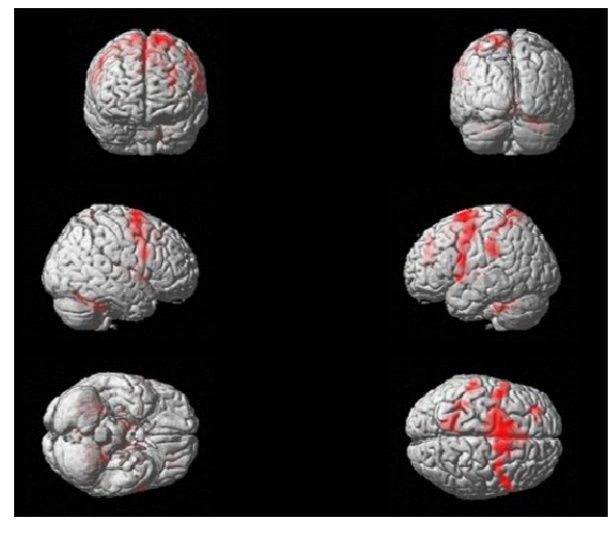

(b)

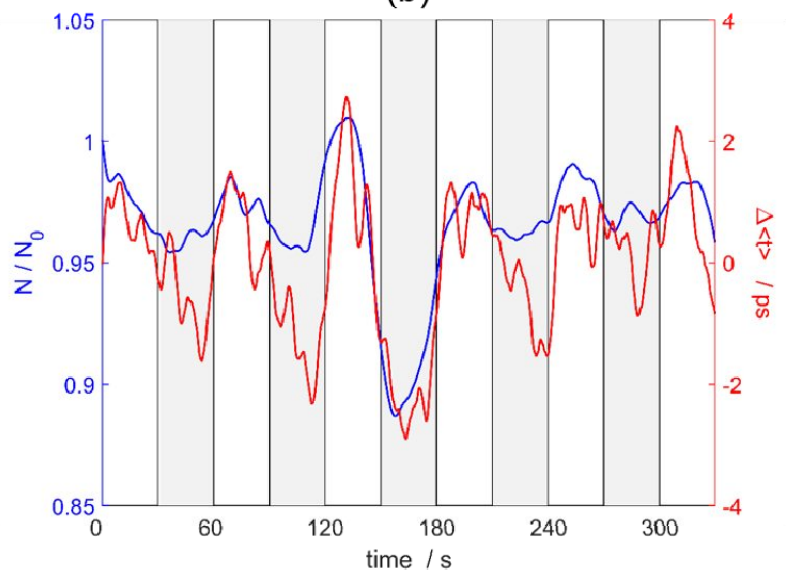

Figure 4. (a) fMRI data obtained from one subject during motor imagery laid over the single subject render template in SMP8. (b) Normalized change in the number of photons $N$ in blue and the change in mean time $\Delta<t>$ in red from one channel obtained during motor imagery and rest and plotted against time. The $N$ signals obtained were normalized by the initial value $N_{0}$ (mean value of $N$ for the first $10 \mathrm{~s}$ ). The grey boxes indicate the periods of motor imagery.

\section{DISCUSSION}

It is well known that motor imagery activates the SMA and PMC, as these regions are involved in imagining and executing coordinated movements ${ }^{22}$. Using this concept, command following by regulating brain activity can not only be used as a tool to assess consciousness, but it can also be used to communicate with patients suffering from disorders of consciousness $^{1,23}$. The goal of this study was to assess the feasibility of a portable, non-invasive, TR-fNIRS system to detect brain activity during motor imagery.

Looking at the phantom stability measurements (presented in Figure 2), the stability of the signals can be clearly observed. The fluctuations in the signals are small, with the noise contamination increasing for higher statistical moments. This is expected since higher moments are more sensitive to late arriving photons, which are small in number. Figure 2 also presents the in vivo stability measurements that show increased variability due to additional physiological noise. It is clear from the results presented in Figure 3 that the mean time and variance are less sensitive to fast frequencies (i.e. heart rate). This could be due to the fact that the higher moments are not as sensitive to changes in the extracerebral layer, and therefore superficial arterial pulsation are not observed in these signals.

Figure 4 shows the fMRI and fNIRS results obtained from one healthy subject during tennis imagery. During the task, the increase in regional blood flow and blood volume in the SMA and/or PMC leads to an increase in light absorption. As a result, there is a decrease in the light being detected and thus a decrease in the $N$ and $\langle t\rangle$. Hence, brain activity during motor imagery is detectable using both the $N$ and $\langle t\rangle$, with comparable contrast to noise ratio for both signals. However, the $\langle t\rangle$ is more sensitive to late arriving photons that have a higher probability of reaching the brain, and thus the $\langle t\rangle$ signal does not suffer as much from extracerebral contamination as the $N$. For the $V$, the signal changes 
associated with brain activity was masked by the high noise present in the signal. Similar fNIRS results were obtained for 3 of the 4 subjects who showed fMRI activity, with decreases in the $N$ and the $<t>$ during each task interval. As for the remaining subject, the $N$ and $\langle t>$ increased during the task, which suggests inverse oxygenation. Similar results were previously reported by Holper et $\mathrm{al}^{24}$ and it was suggested that inverse oxygenation is correlated with the task mode (i.e. motor execution or motor imagery) and the task complexity (simple versus complex tasks). However, the fMRI timecourse for this subject was similar to the other subjects and did not indicate inverse oxygenation, and hence this result warrants further investigation. As for the 3 subjects with no detectable fMRI or fNIRS activity, this is not uncommon and similar results have been reported previously in the literature ${ }^{5}$.

Although the results obtained are promising and show good agreement between the two imaging modalities, additional experiments are required to characterize the sensitivity of the first three moments for detecting brain activity. Motor imagery experiments will also be repeated using short task periods $(\sim 10 \mathrm{~s})$. This is relevant for BCI applications in which real time communication is ultimately the goal, and thus repeated $30 \mathrm{~s}$ task blocks is too slow.

In conclusion, this preliminary study highlights the potential of time-resolved fNIRS in detecting brain activity during motor imagery. Having a safe and portable system for detecting brain activity could provide a tool for assessing awareness at the bedside, which can set the stage for future studies involving patients with disorders of consciousness.

\section{ACKNOWLEDGMENTS}

This study was funded by the Canada Excellence Research Chair (CERC) program in Cognitive Neuroscience and Imaging and the Natural Sciences and Engineering Research Council of Canada (NSERC). The authors would also like to acknowledge Mr. Lynn Keenlyside for his assistance in building components for the TR-NIRS system as well as printing the fNIRS holder used in this study.

\section{REFERENCES}

[1] Monti, M.M., Vanhaudenhuyse, A., Coleman, M.R., Boly, M., Pickard, J.D., Tshibanda, L., Owen, A.M., and Laureys, S., "Willful modulation of brain activity in disorders of consciousness.," N Engl J Med 362, 579-589 (2010).

[2] Cruse, D., Chennu, S., Chatelle, C., Bekinschtein, T.A., Fernández-Espejo, D., Pickard, J.D., Laureys, S., and Owen, A.M., "Bedside detection of awareness in the vegetative state: A cohort study," The Lancet 378, 2088 2094 (2011).

[3] Naci, L., Monti, M.M., Cruse, D., Kübler, A., Sorger, B., Goebel, R., Kotchoubey, B., and Owen, A.M., "Braincomputer interfaces for communication with nonresponsive patients," in Ann. Neurol. 72, pp. 312-323 (2012).

[4] Owen, A.M., Coleman, M.R., Boly, M., Davis, M.H., Laureys, S., and Pickard, J.D., "Detecting Awareness in the Vegetative State," Science 313(5792), 1402-1402 (2006).

[5] Fernández-Espejo, D., Norton, L., and Owen, A.M., "The clinical utility of fMRI for identifying covert awareness in the vegetative state: a comparison of sensitivity between 3T and 1.5T.," PloS one 9(4), e95082 (2014).

[6] Laureys, S., and Schiff, N.D., "Coma and consciousness: Paradigms (re)framed by neuroimaging," NeuroImage 61(2), 478-491 (2012).

[7] Boly, M., Coleman, M.R., Davis, M.H., Hampshire, A., Bor, D., Moonen, G., Maquet, P.A., Pickard, J.D., Laureys, S., et al., "When thoughts become action: An fMRI paradigm to study volitional brain activity in noncommunicative brain injured patients," NeuroImage 36, 979-992 (2007).

[8] Gibson, R.M., Fernández-Espejo, D., Gonzalez-Lara, L.E., Kwan, B.Y., Lee, D.H., Owen, A.M., and Cruse, D., "Multiple tasks and neuroimaging modalities increase the likelihood of detecting covert awareness in patients with Disorders of Consciousness," Frontiers in Human Neuroscience 8(950), 1-9 (2014).

[9] Benaron, D.A., Hintz, S.R., Villringer, A., Boas, D., Kleinschmidt, A., Frahm, J., Hirth, C., Obrig, H., van Houten, J.C., et al., "Noninvasive functional imaging of human brain using light.," Journal of cerebral blood flow and metabolism : official journal of the International Society of Cerebral Blood Flow and Metabolism 20(3), 469-477 (2000).

[10] Re, R., Contini, D., Turola, M., Spinelli, L., Zucchelli, L., Caffini, M., Cubeddu, R., and Torricelli, A., "Multichannel medical device for time domain functional near infrared spectroscopy based on wavelength space 
multiplexing.," Biomedical optics express 4, 2231-46 (2013).

[11] Torricelli, A., Contini, D., Pifferi, A., Caffini, M., Re, R., Zucchelli, L., and Spinelli, L., "Time domain functional NIRS imaging for human brain mapping," in Neuroimage 85, pp. 28-50 (2014).

[12] Elliott, J.T., Diop, M., Tichauer, K.M., Lee, T.-Y., and Lawrence, K. St., "Quantitative measurement of cerebral blood flow in a juvenile porcine model by depth-resolved near-infrared spectroscopy," in J. Biomed. Opt. 15, p. 037014 (2010).

[13] Milej, D., Janusek, D., Gerega, A., Wojtkiewicz, S., Sawosz, P., Treszczanowicz, J., Weigl, W., and Liebert, A., "Optimization of the method for assessment of brain perfusion in humans using contrast-enhanced reflectometry: multidistance time-resolved measurements," Journal of Biomedical Optics 20(10), 106013 (2015).

[14] Farina, A., Torricelli, A., Bargigia, I., Spinelli, L., Cubeddu, R., Foschum, F., Jäger, M., Simon, E., Fugger, O., et al., "In-vivo multilaboratory investigation of the optical properties of the human head," Biomed. Opt. Express 6(7), 2609-2623 (2015).

[15] Steinbrink, J., Wabnitz, H., Obrig, H., Villringer, A., and Rinneberg, H., "Determining changes in NIR absorption using a layered model of the human head," Phys Med Biol 46(3), 879-896 (2001).

[16] Diop, M., and St Lawrence, K., "Improving the depth sensitivity of time-resolved measurements by extracting the distribution of times-of-flight.," Biomedical optics express 4(3), 447-59 (2013).

[17] Milej, D., Gerega, A., Kacprzak, M., Sawosz, P., Weigl, W., Maniewski, R., and Liebert, A., "Time-resolved multi-channel optical system for assessment of brain oxygenation and perfusion by monitoring of diffuse reflectance and fluorescence," Opto-Electronics Review 22(1), 55-67 (2014).

[18] Liebert, A., Wabnitz, H., Grosenick, D., Möller, M., Macdonald, R., and Rinneberg, H., "Evaluation of optical properties of highly scattering media by moments of distributions of times of flight of photons.," Applied optics 42(28), 5785-5792 (2003).

[19] Milej, D., Abdalmalak, A., Janusek, D., Diop, M., Liebert, A., and Lawrence, K. St., "Time-resolved subtraction method for measuring optical properties of turbid media," Appl. Opt. 55(7), 1507-1513 (2016).

[20] Verdecchia, K., Diop, M., Morrison, L.B., Lee, T.-Y., and Lawrence, K. St., "Assessment of the best flow model to characterize diffuse correlation spectroscopy data acquired directly on the brain," Biomed. Opt. Express 6(11), 4288-4301 (2015).

[21] Wabnitz, H., Jelzow, A., Mazurenka, M., Steinkellner, O., Macdonald, R., Milej, D., Zolek, N., Kacprzak, M., Sawosz, P., et al., "Performance assessment of time-domain optical brain imagers, part 2: nEUROPt protocol.," Journal of biomedical optics 19(8), 86012 (2014).

[22] Owen, A.M., "When thoughts become actions: functional neuroimaging in the vegetative state," Future Neurology 1(6), 693-695 (2006).

[23] Fernández-Espejo, D., and Owen, A.M., "Detecting awareness after severe brain injury.," Nature reviews. Neuroscience 14, 801-9 (2013).

[24] Holper, L., Shalóm, D.E., Wolf, M., and Sigman, M., "Understanding inverse oxygenation responses during motor imagery: A functional near-infrared spectroscopy study," European Journal of Neuroscience 33, 2318 2328 (2011). 